\title{
Resonant, non-resonant, and anomalous states of Dirac electrons in a parabolic well in the presence of magnetic fields
}

\author{
S. C. Kim, J. W. Lee, and S. -R. Eric Yang* \\ Physics Department, Korea University, Seoul Korea 136-713 \\ E-mail: *corresponding author eyang812@gmail.com
}

\begin{abstract}
We report on several new basic properties of a parabolic dot in the presence of a magnetic field. The ratio between the potential strength and the Landau level (LL) energy spacing serves as the coupling constant of this problem. In the weak coupling limit the energy spectrum in each Hilbert subspace of an angular momentum consists of discrete LLs of graphene. In the intermediate coupling regime non-resonant states form a closely spaced energy spectrum. We find, counterintuitively, that resonant quasi-boundstates of both positive and negative energies exist in the spectrum. The presence of resonant quasi-boundstates of negative energies is a unique property of massless Dirac fermions. As the strong coupling limit is approached resonant and non-resonant states transform into anomalous states, whose probability densities develop a narrow peak inside the well and another broad peak under the potential barrier. These properties may investigated experimentally by measuring optical transition energies that can be described by a scaling function of the coupling constant.
\end{abstract}

\section{Introduction}

Two-dimensional parabolic quantum dots of semiconductor heterostructures have been studied widely [1, 2] both experimentally and theoretically because they are excellent candidates for single electron transistors. They can effectively confine electrons and the number of electrons in them can be controlled using a gate potential. Their Hamiltonian is

$$
H=\frac{1}{2 m}\left(\vec{p}+\frac{e}{c} \vec{A}\right)^{2}+\frac{1}{2} m \Omega^{2} r^{2}
$$

with a magnetic field $\vec{B}$ is applied perpendicular to the 2D plane (vector potential $\vec{A}$ is given in a symmetric gauge). The characteristic length scale of the problem is given by $\lambda^{2}=\frac{\hbar}{m \sqrt{4 \Omega^{2}+\omega_{c}^{2}}}$, where $\omega_{c}=\frac{e B}{m c}$ is the cyclotron frequency. This problem can be solved exactly[3], and the eigenenergies are all positive and their spectrum is discrete.

Massless Dirac electrons [4] moving in a 2D parabolic potential display several different features in comparison to massful electrons. They are described by the Dirac 
Resonant, non-resonant, and anomalous states of Dirac electrons in a parabolic well in the presence of magn Hamiltonian

$$
H=v_{F} \vec{\sigma} \cdot\left(\vec{p}+\frac{e}{c} \vec{A}\right)+\frac{1}{2} \kappa r^{2} .
$$

No exact solutions of this problem are known in graphene and several fundamental properties are still unknown, such as the existence of resonant and non-resonant states. These basic properties may affect the experimentally relevant optical spectrum in a profound way. The dimensionless coupling constant of this problem is the ratio between the strength of the potential $\frac{1}{2} \kappa \ell^{2}$ and the LL energy separation $E_{C}=\frac{\hbar v_{F}}{\ell}$

$$
\alpha=\kappa \ell^{2} / E_{C}=\frac{\kappa \ell^{3}}{\hbar v_{F}},
$$

where the magnetic length is $\ell=\sqrt{\hbar c / B e}$. One is in the strong coupling regime $\alpha \gg 1$ for small value of $B$ or large value of potential strength $\kappa$. Parabolic dots in magnetic fields have been investigated numerically in the weak coupling regime $\alpha<1$. The energy spectrum is found to be discrete, and eigenstates are quasi-boundstates with long oscillating tails under the barrier [5, 6, 7]. Also some of these states exhibit anticrossings [5, 7]. The parabolic potential acts as a singular perturbation [8 because eigenstate wavefunctions are qualitatively different from those in the absence of a parabolic potential.

The problem has not been investigated away from the weak coupling regime. It is a highly non-trivial problem. This can be seen as follows. One of the special features of graphene LLs is the presence of negative energy states under the potential barrier[7]. The first order energy correction of a LL state $\psi_{n, m}(r)$ is, for sufficiently large $n$,

$$
\left\langle\psi_{n, m}|V(r)| \psi_{n, m}\right\rangle \sim \kappa\left\langle r^{2}\right\rangle \sim \kappa \ell^{2}|n| .
$$

This result suggests that a LL state with a large negative energy, $-E_{C} \sqrt{2|n|}$ with $|n| \gg 1$, corresponding to having a large average radius $\sqrt{\left\langle r^{2}\right\rangle}$, acquires a significant positive energy correction, which can make the renormalized energy positive. In the dimensionless units this energy correction is $\kappa \ell^{2}|n| / E_{C}=\alpha|n|$, which suggests that even for small value of $\alpha$ the correction can be significant for $|n| \gg 1$. Moreover, it is unclear how eigenstates evolve from weak to strong coupling regimes. A simple dimensional analysis suggests that the energy scale of the problem in the strong coupling limit of $B \rightarrow 0$ or $\alpha \rightarrow \infty$ is $\kappa^{1 / 3}\left(\hbar v_{F}\right)^{2 / 3}$. In units of $E_{C}$ this energy scale is $\alpha^{1 / 3}$. It indicates that the dimensionless energy level spacing increases from $\sim 1$ to $\sim \alpha^{1 / 3}$ as one moves from weak to strong coupling regimes. However, studies in ordinary semiconductors suggest that the crossover regime may be non-trivial [9].

We have investigated these issues by solving large Hamiltonian matrices. Let us give a brief summary of our results in a Hilbert subspace of angular momentum $J$. We have studied how eigenvalues and eigenstates evolve as $\alpha$ increase and find that they change in a non-trivial way. In the weak coupling limit of $\alpha \rightarrow 0$ the spectrum consists of discrete LLs. In the intermediate coupling regime $\alpha \sim 1$ non-resonant states form a closely spaced energy spectrum, see Fig,1. We find, counter-intuitively, that 
Resonant, non-resonant, and anomalous states of Dirac electrons in a parabolic well in the presence of magn

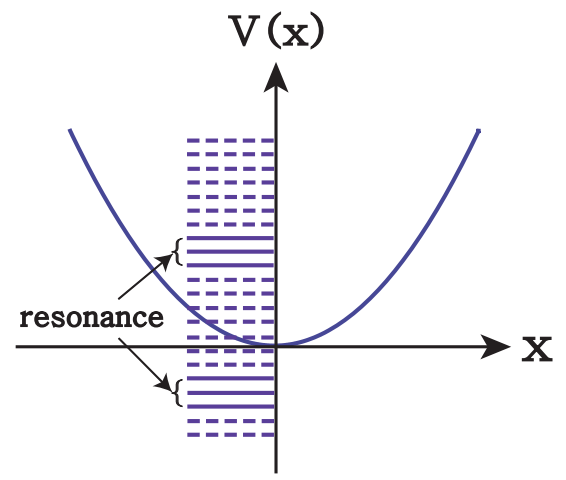

Figure 1. Schematic energy spectum of a parabolic dot in the intermediate coupling regime $\alpha \sim 1$. Energy spectrum is closely spaced, and resonant states of positive and negative energies are present.

resonant quasi-boundstates of both positive and negative energies exist. The presence of resonant quasi-boundstates of negative energies is a unique property of massless Dirac fermions, but they are well-defined only for $\alpha<1$. In the strong coupling regime $\alpha \gg 1$ both resonant and non-resonant states transform into anomalous states, and a sharp distinction between resonant and non-resonant states no longer exists. Probability densities of anomalous states develop a narrow peak inside the well and decays slowly with small oscillations under the barrier. The energy level spacing between them is proportional to the value $\kappa^{1 / 3}\left(\hbar v_{F}\right)^{2 / 3}$ and is independent of $\ell$. We show that optical transition energies between resonant quasi-boundstates can be described by a scaling function of $\alpha$.

\section{Basis states and Hamiltonian matrix}

In our Hamiltonian matrix approach the basis states are chosen as graphene LL states $\psi_{n, m}(\vec{r})$ with two components $\mathrm{A}$ and $\mathrm{B}$

$$
\psi_{n, m}(\vec{r})=c_{n}\left(\begin{array}{c}
-\operatorname{sgn}(n) i \phi_{|n|-1, m}(\vec{r}) \\
\phi_{|n|, m}(\vec{r})
\end{array}\right) .
$$

Here $\operatorname{sgn}(n)=-1,0,1$ for $n<0, n=0, n>0$ with $n$ and $m$ integers $(m \geq 0)$, and $c_{n}=1$ for $n=0$ and $1 / \sqrt{2}$ otherwise. These basis states can have positive or negative LL energies:

$$
E_{n}=\operatorname{sgn}(n) E_{C} \sqrt{2|n|} .
$$

The wavefunctions $\phi_{n, m}(\vec{r})$ are the Landau level wavefunctions of ordinary twodimensional systems [10]

$$
\begin{aligned}
\phi_{n, m}(\vec{r}) & =A_{n, m} \exp \left(i(n-m) \theta-\frac{r^{2}}{4 \ell^{2}}\right)\left(\frac{r}{\ell}\right)^{|m-n|} \\
& \times L_{(n+m-|m-n|) / 2}^{|m-n|}\left(\frac{r^{2}}{2 \ell^{2}}\right),
\end{aligned}
$$


Resonant, non-resonant, and anomalous states of Dirac electrons in a parabolic well in the presence of magn

where $A_{n, m}$ are the normalization constants and $L_{n}^{m}(x)$ are Laguerre polynomials. In the presence of a parabolic potential $J=|n|-m-\frac{1}{2}$ remains a good quantum number. The average radius of $\phi_{n, m}(\vec{r})$ is given by

$$
\left\langle r^{2}\right\rangle=2 \ell^{2}(n+m+1)=2 \ell^{2}(n+|n|-J+1 / 2) .
$$

To investigate the strong coupling effects a large number of basis states $\psi_{n, m}(r)$ is required. It is convenient to divide the Hilbert space into subspaces of angular momentum $J= \pm \frac{1}{2}, \pm \frac{3}{2}, \pm \frac{5}{2}, \cdots$. We diagonalize the Hamiltonian matrix in each Hilbert subspace $J$. For given $J$, the matrix elements of the parabolic potential can be written as sum of two components:

$$
\begin{aligned}
& \left\langle\psi_{n, m}\left|\frac{V(r)}{E_{C}}\right| \psi_{n^{\prime}, m^{\prime}}\right\rangle \\
& =c_{n} c_{n^{\prime}} \operatorname{sgn}\left(n n^{\prime}\right)\left\langle\phi_{|n|-1, m}\left|\frac{V(r)}{E_{C}}\right| \phi_{\left|n^{\prime}\right|-1, m^{\prime}}\right\rangle \\
& \quad+c_{n} c_{n^{\prime}}\left\langle\phi_{|n|, m}\left|\frac{V(r)}{E_{C}}\right| \phi_{\mid n^{\prime}, m^{\prime}}\right\rangle .
\end{aligned}
$$

Using the following property of Laguerre polynomials

$$
L_{n}^{\alpha}(x)=\frac{1}{x}\left[(n+\alpha+1) L_{n}^{\alpha-1}(x)-(n+1) L_{n+1}^{\alpha-1}(x)\right],
$$

and the orthogonality

$$
\int_{0}^{\infty} x^{\alpha} \mathrm{e}^{-x} L_{n}^{\alpha}(x) L_{m}^{\alpha}(x) \mathrm{d} x=\frac{\Gamma(n+\alpha+1)}{n !} \delta_{n, m},
$$

we evaluate the matrix elements. The resulting matrix is a sparse matrix, see Fig.2. The dimension of the Hamiltonian matrix is denoted by $N_{c}$. When the value of $N_{c}$ is sufficiently large the states investigated in this paper do not exhibit dependence on $N_{c}$.

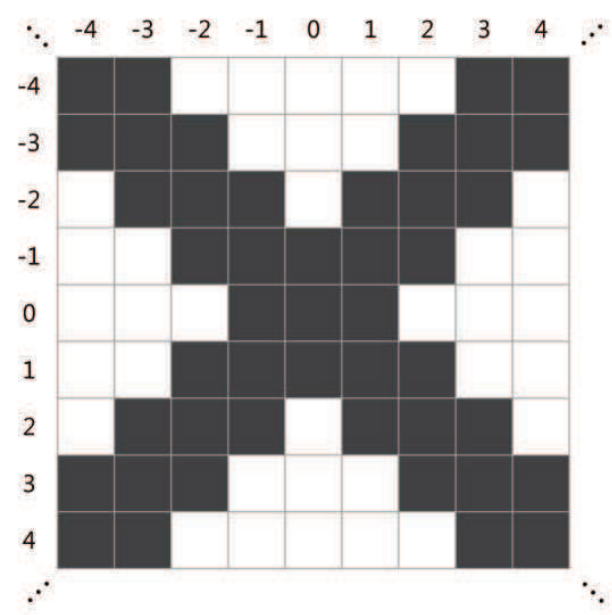

Figure 2. Structure of the Hamiltonian matrix is shown for $J<0$. For a given $J$ we choose to use $n$ as the basis index instead of $m$. It runs from $-\left(N_{c}-1\right) / 2$ to $\left(N_{c}-1\right) / 2$. Filled (empty) squares represent non-zero (zero) elements. For $J>0$ allowed basis states are given in TABLE II of Ref.[11. 
Resonant, non-resonant, and anomalous states of Dirac electrons in a parabolic well in the presence of magn

\section{Eigenstates of a Hilbert subspace}

Eigenstates of a Hilbert subspace are obtained by diagonalizing the Hamiltonian matrix. They may be written as a linear combination of LL wavefunctions with same angular momentum:

$$
\Psi_{N}^{J}(r)=\sum_{n} C_{n} \psi_{n, m}(r)
$$

Here quantum number $N$ is chosen to be the value $n$ for which $\left|C_{n}\right|$ is maximum[12].

The following exact results[11] are useful in checking numerical results. The value of wavefunctions at $r=0$ is non-zero only for $J=-\frac{1}{2}$ and $\frac{1}{2}$ :

$$
\Psi_{N}^{J}(0)=\left\{\begin{array}{ccc}
0 & \text { for } & J \neq \pm 1 / 2 \\
\text { finite } & \text { for } & J= \pm 1 / 2
\end{array}\right.
$$

The B and A components of $\left|\Psi_{N}^{-1 / 2}\right\rangle$ and $\left|\Psi_{N}^{1 / 2}\right\rangle$ are non-zero and are of s-wave type. Their values at $r=0$ can be written as

$$
\begin{aligned}
& \Psi_{N, B}^{-1 / 2}(0)=\sum_{n \neq 0} A_{|n|,|n|} C_{n} / \sqrt{2}+C_{0} A_{0,0} \\
& \Psi_{N, A}^{1 / 2}(0)=-i \sum_{n} \operatorname{sgn}(n) A_{|n|-1,|n|-1} C_{n} / \sqrt{2} .
\end{aligned}
$$

We will only concentrate on optical transitions involving states with $J=1 / 2,-1 / 2$, or $-3 / 2$ since they give the strongest optical strengths. Unless stated otherwise the results reported in this paper are for the potential strength $\kappa=0.1 \mathrm{meV} / \mathrm{nm}^{2}$.

\subsection{Resonant quasi-boundstates}

The computed energy spectrum of $J=-1 / 2$ is shown in Fig. $3(a)$ for the range $0.029<\alpha<0.47(3.14 T<B<20 T)$. Spectra for other values of $J=1 / 2$ and $-3 / 2$ are similar, as shown in Sec.IV. Lines labeled by $N$ represent the energies of resonant quasi-boundstates and other lines represent non-resonant states. As shown in Fig](b), in the intermediate coupling regime $\alpha \sim 1$, the energy levels are closely spaced due to negative energy LL states whose energies get strongly perturbed upward by the parabolic potential, see Eq.(4). A resonant quasi-boundstate anticrosses other states and becomes strongly mixed with the adjacent states, see Fig.3(b). For example, the resonant quasi-boundstate $\left|\Psi_{0}^{-1 / 2}\right\rangle$ is strongly mixed[12] at $B=3.14 \mathrm{~T}(\alpha=0.47)$, and, as shown in Fig.3(b), there are three states that could be identified as $\left|\Psi_{0}^{-1 / 2}\right\rangle$. In these states with the energies $E=0.025,0.019$, and $0.010 \mathrm{eV}$ the expansion coefficients $C_{n}$ of Eq.(12) take the maximum value at $n=0$ with the values $C_{0}=0.622,0.595$, and 0.287 , respectively (Since $C_{0}$ is largest for $E=0.025 \mathrm{eV}$ this state is labeled as $\left|\Psi_{0}^{-1 / 2}\right\rangle$ ). Fig] (c) displays probability densities of these states. They form together a resonance with the approximate resonant energy $E=0.025 \mathrm{eV}$. In the weak coupling regime the width of a resonance is small and, in order to resolve it, the energy level spacing must be smaller than the width of a resonance, which requires a large matrix dimension. 
Resonant, non-resonant, and anomalous states of Dirac electrons in a parabolic well in the presence of magn
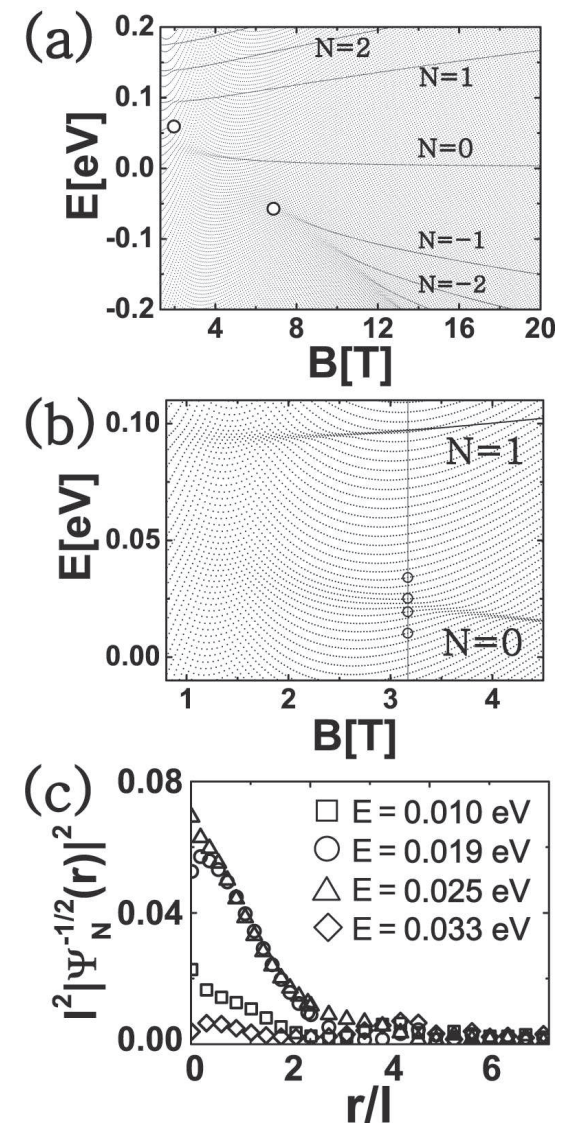

Figure 3. (a) Eigenenergy spectrum of Hilbert subspace of $J=-\frac{1}{2}$. Lines labeled by $N$ represent resonant quasi-boundstates. $2001 \times 2001$ matrix is used. (b) Enlarged energy spectrum for $J=-\frac{1}{2}$. Resonant quasi-boundstate $\left|\Psi_{0}^{-1 / 2}\right\rangle$ anticrosses strongly at $B=3.14 \mathrm{~T}(\alpha=0.47)$. Four circles represent these coupled states. (c) Probability densities of these four states are displayed. They form together a resonance with the approximate resonant energy $\epsilon_{0}^{-1 / 2}(3.14)=0.025 \mathrm{eV}$.

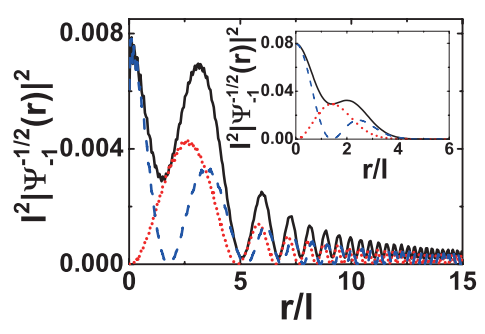

Figure 4. An example of a resonant quasi-boundstate of negative energy (indicated as a open circle in Fig 3(a)). Dotted (dashed) line represents A (B) components of the probability density of $\left|\Psi_{N}^{J}\right\rangle=\left|\Psi_{-1}^{-1 / 2}\right\rangle$ with energy $E=-0.060 \mathrm{eV}$ computed at the coupling constant $B=6.88 \mathrm{~T}(\alpha=0.145)$. Solid line is the total probability density. $2001 \times 2001$ matrix is used. In the absence of the potential the wavefunction has a peak near $r=0$ (see inset), and its energy is negative $E=-\sqrt{2} E_{C}=-0.093 \mathrm{eV}$. 
Resonant, non-resonant, and anomalous states of Dirac electrons in a parabolic well in the presence of magn

Note also that resonant quasi-boundstates of negative energies exist. This is a unique property of massless Dirac fermions. An example is shown in Fig, 4. They are well-defined only for sufficiently large $B$, i.e., only in the weak coupling regime. The appearance of a second peak away from $r=0$ in the probability density is different from the usual behavior of the wavefunction a resonant state.

We see that as $\alpha$ increases, or, as $B$ decreases, resonant quasi-boundstates disappear into the closely spaced energy spectrum. For $N=0$ states this happens around $B \sim 3 T$. For larger values of $N$ this happens at smaller values of $B$. We will show in Sec.III (C) that, as $\alpha$ increases, the peak at $r=0$ increases and the state becomes anomalous.

\subsection{Non-resonant states}

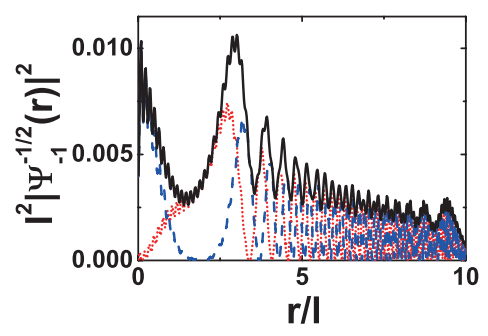

Figure 5. An example of a non-resonant state (indicated as a open circle in Fig 3(a)). Dotted (dashed) line represents A (B) components of the probability density $\left|\Psi_{N}^{J}\right\rangle=\left|\Psi_{-1}^{-1 / 2}\right\rangle$ with energy $E=0.054 \mathrm{eV}$ and $B=2.04 \mathrm{~T}(\alpha=0.9)$. As $r \rightarrow 0$ the dashed line approaches a finite value while dotted line goes to zero. $2001 \times 2001$ matrix is used.

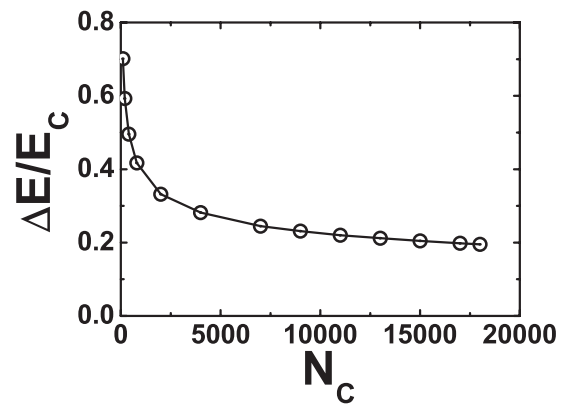

Figure 6. Average energy level spacing of non-resonant states near $E=0$ as a function of $N_{c}$ at $B=1.9 \mathrm{~T}(\alpha=1)$. The dimension $N_{c}$ varies from 101 to 18001 . Matrix sizes $N_{c}$ are 101, 201, 401, 801, 2001, 4001, 9001, 11001, 15001, and 18001.

The energy spectrum of $J=-1 / 2$ in Fig.3(a) also display non-resonant states. Probability density of a non-resonant state looks qualitatively different from that of the corresponding unrenormalized LL state. The probability density of a non-resonant state $\left|\Psi_{-1}^{-1 / 2}(r)\right|^{2}$ at $B=2.04 \mathrm{~T}(\alpha=0.9)$ is shown in Fig.5(a). Its wavefunction has a large peak at $r=0$, which is different from the usual behavior of the wavefunction 
Resonant, non-resonant, and anomalous states of Dirac electrons in a parabolic well in the presence of magn

a non-resonant state. As $\alpha$ increases the peak at $r=0$ increases even more and the state becomes anomalous. Non-resonant states are unique to graphene parabolic wells and do not exist in ordinary parabolic wells. In the absence of the parabolic potential its energy is $E=-\sqrt{2} E_{C}=-0.051 \mathrm{eV}$ while in the presence of the potential it is $\epsilon_{-1}^{-1 / 2}(2.04)=1.5 E_{C}=0.054 \mathrm{eV}$. Fig 6 displays the energy level spacing of non-resonant states as a function of $N_{c}$ at $\alpha=1(B=1.9 \mathrm{~T})$. We observe that the level spacing decreases rather slowly for large $N_{c}$. However, the energies of resonant quasi-boundstate converge rather quickly, see Fig.9.

\subsection{Anomalous states}
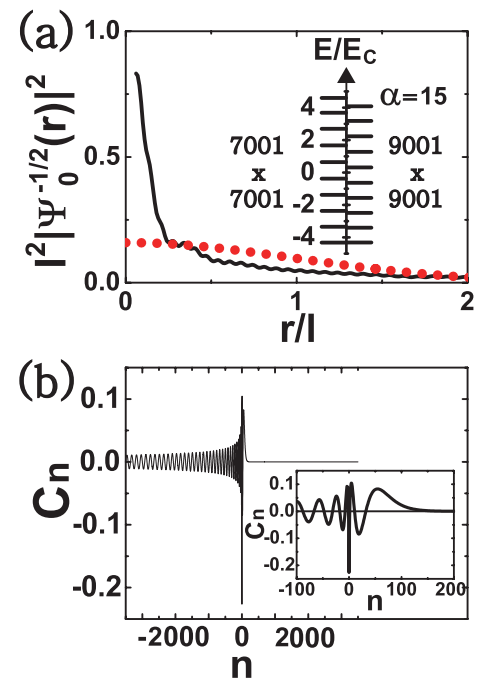

Figure 7. (a) Total probability density of $\left|\Psi_{0}^{-1 / 2}\right\rangle$ with energy $0.177 \mathrm{eV}$ at $B=0.312 \mathrm{~T}$ ( $\alpha=15$ ) (solid). Corresponding state at $\alpha=0$ is shown as dotted line. The eigenstate is obtained by diagonalizing $7001 \times 7001$ Hamiltonian matrix. Energy levels are also shown at $B=0.312 \mathrm{~T}$ ( $\alpha=15$ ) for $N_{c}=7001$ and 9001. (b) Expansion coefficients $C_{n}=\left\langle n \mid \Psi_{0}^{-1 / 2}\right\rangle$ of anomalous eigenstate $\left|\Psi_{0}^{-1 / 2}\right\rangle$.

The energy spectrum of $J=-1 / 2$ in Fig.3(a) also display anomalous states at low magnetic fields of the strong coupling regime $\alpha \gg 1$. In this regime both resonant and non-resonant states transform into anomalous states, and a sharp distinction between resonant and non-resonant states no longer exists. Such a state is shown in Fig.7(a) with the energy $E=0.177 \mathrm{eV}$ (in unit of $E_{C}$ it is 12.6). We see in $\operatorname{Fig}: 7$ (a) that the peak value of probability density at $r=0$ is much larger than the unperturbed value of $\frac{1}{2 \pi} \simeq 0.16$. [13]. For this state the penetration into the barrier should start from the turning point $r_{b}$ satisfying $\frac{1}{2} \kappa r_{b}^{2}=E$. From this we find that the value $r_{b} / \ell$ is 1.3 , which is rather different from the estimate of about 0.3 obtained from numerical result shown in Fig:7(a) (note that the probability density under the barrier oscillates). An anomalous state is a strong coupling effect and can only be obtained correctly by computing large Hamiltonian matrices. 
Resonant, non-resonant, and anomalous states of Dirac electrons in a parabolic well in the presence of magn

Fig.7(b) displays the expansion coefficient $C_{n}$ as a function of $n$. Note that $C_{n}$ has a long oscillating tail for $n<0$. This is intimately related to the probability density having a long oscillating tail under the barrier. The sum of $C_{n}$ for $n<0$ is approximately zero while the sum for $n \geq 0$ is finite and makes $\Psi_{0}^{-1 / 2}(0)$ large (see Eq.(14)). Note that the probability density under the barrier is somewhat smaller than that of $\alpha=0$.

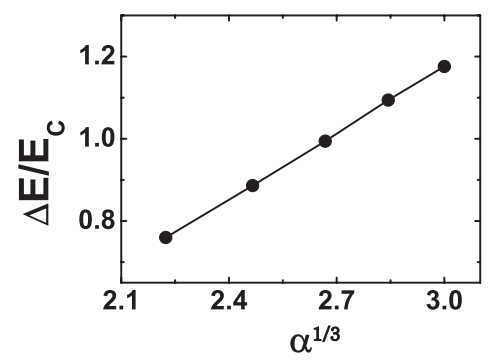

Figure 8. Dimensionless average energy level spacing of the energy spectrum of Hilbert subspace $J=-1 / 2$ in the strong coupling regime. We have used $N_{c}=9001$.

When $B \rightarrow 0$ or $\alpha \rightarrow \infty$ the natural length and energy scales of the problem are $\xi=\left(\frac{\hbar v_{F}}{\kappa}\right)^{1 / 3}$ and $\kappa^{1 / 3}\left(\hbar v_{F}\right)^{2 / 3}$ (Note $\xi / \ell=\alpha^{-1 / 3}$ ). In units of $E_{C}$ this energy scale is $\alpha^{1 / 3}$, which should be proportional to the dimensionless energy level spacing of the Hilbert subspace of $J$ in the strong coupling regime. Our numerical results in the strong coupling regime $\alpha \gg 1$ are indeed consistent with this, see Fig, 8 ,

\section{Scaling of optical transitions}

\subsection{Scaling results}

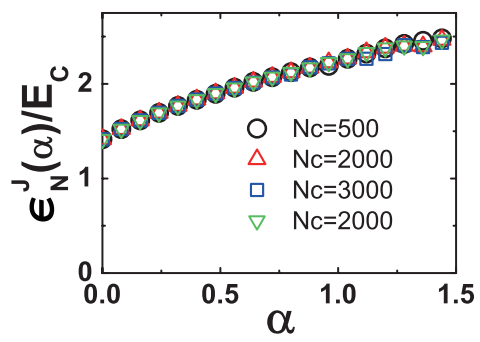

Figure 9. Data collapse of dimensionless energies of quasi-boundstate $\epsilon_{1}^{1 / 2}(\alpha)$ for different values of $N_{c}$ and $\kappa$. Circles, triangles, and squares are for $\kappa=0.1 \mathrm{meV} / \mathrm{nm}^{2}$. Inverted tiangles are for $\kappa=0.2 \mathrm{meV} / \mathrm{nm}^{2}$.

In the previous section we showed that energies of the resonant quasi-boundstates depend on both $\kappa$ and $B$. Here we will show that their energies, when measured in units of $E_{C}$ in the limit of large $N_{c}$, follow a scaling function of a single dimensionless variable, namely, the dimensionless coupling constant $\alpha$, see Fig.9, Fig.10(a) displays the dimensionless energies of resonant quasibound states $\epsilon_{N}^{J}(\alpha)$ as a function of $\alpha$ for $J=-1 / 2$ and $1 / 2$. Fig $[10$ (b) displays similar results for $J=-3 / 2$ and $-1 / 2$. 
Resonant, non-resonant, and anomalous states of Dirac electrons in a parabolic well in the presence of magn
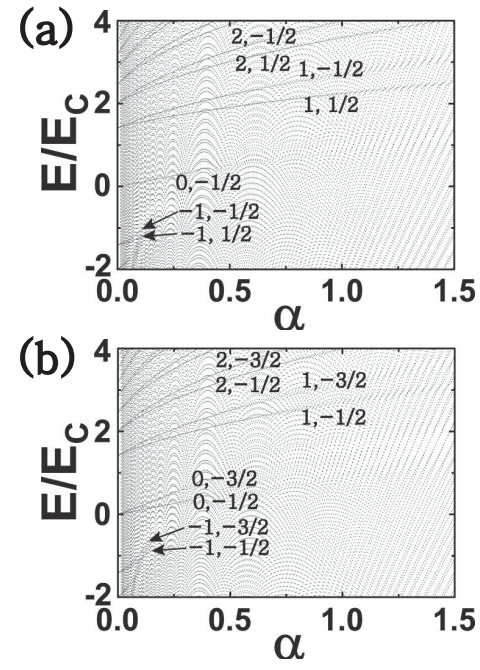

Figure 10. (a) Energy spectra for $J=-\frac{1}{2}$ and $\frac{1}{2}$ plotted together. (b) Energy spectra for $J=-\frac{1}{2}$ and $-\frac{3}{2}$ plotted together. Matrix sizes $N_{c}$ are 2001 for $J=-\frac{1}{2}, 2001$ for $J=-\frac{3}{2}$, and 2000 for $J=\frac{1}{2}$.

Since dimensionless energies of resonant quasi-boundstates $\epsilon_{N}^{J}(\alpha)$ satisfy a scaling function the transition energies $E=\epsilon_{N^{\prime}}^{J^{\prime}}(\alpha)-\epsilon_{N}^{J}(\alpha)$ between them also obey a scaling

$$
\frac{E}{E_{C}}=f_{N \rightarrow N^{\prime}}^{J \rightarrow J^{\prime}}(\alpha) .
$$

This scaling relation holds as long as quasi-boundstates are well defined.

\subsection{Optical transition energies and selection rules}

Before we compute strengths and selection rules of optical transitions let us first mention some useful results in computing them. First, in the absence of a parabolic potential absorption selection rules are $\epsilon_{N}^{-1 / 2}(\alpha) \rightarrow \epsilon_{N+1}^{1 / 2}(\alpha)$ for $N \geq 0$ and $\epsilon_{N}^{1 / 2}(\alpha) \rightarrow \epsilon_{N+1}^{-1 / 2}(\alpha)$ for $N<0$. These selection rules are displayed schematically in Fig.11 (see also TABLE II in Ref.[11]). Due to mixing of different LL states by the parabolic potential the selection rule $\Delta N= \pm 1$ must be relaxed. Second, the energies of the $N=0 \mathrm{LL}$ states are split by the perturbing parabolic potential and they increase as $J$ decreases: $\epsilon_{0}^{-1 / 2}(\alpha)<\epsilon_{0}^{-3 / 2}(\alpha)<\epsilon_{0}^{-5 / 2}(\alpha)<\cdots$. This is also true for other LL states. Third, the optical transitions with the strongest strength occur between $J=1 / 2$ and $-1 / 2$. Other transitions, for example, transitions between $J=-3 / 2$ and $-1 / 2$ are weaker: the transition $\epsilon_{0}^{-1 / 2}(0.25) \rightarrow \epsilon_{1}^{1 / 2}(0.25)$ has the strength 0.37 with transition energy $1.44 E_{C}$ while the transition $\epsilon_{0}^{-3 / 2}(0.25) \rightarrow \epsilon_{1}^{-1 / 2}(0.25)$ has the strength 0.21 with transition energy $1.34 E_{C}$ (the involved energy levels are shown in Fig 10). These strengths and the corresponding transition energies decrease as the value $J$ of initial states of optical transitions decreases.

Let us use these results to understand what type of optical transitions are possible. For this purpose we display in Fig 12, for a relatively small value of $N_{c}$, eigenenergies 
Resonant, non-resonant, and anomalous states of Dirac electrons in a parabolic well in the presence of magn

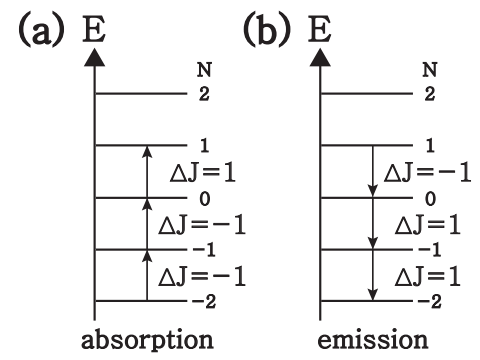

Figure 11. We assume that photons are polarized along x-axis. (a) Some examples of absorption selection rules for LL states in the absence of a parabolic potential given by Eq.(5). In fact there are also transitions with the selection rule $N \rightarrow|N|+1$ with $\Delta J=1(N \leq-1)$ and $N \rightarrow|N|-1$ with $\Delta J=-1(N \leq-2)$. However, they have high energies and are not considered here. (b) Some examples of emission selection rules are shown. Selection rules $N \rightarrow-|N|-1$ with $\Delta J=1(N \geq 1)$ and $N \rightarrow-|N|+1$ with $\Delta J=-1(N \geq 2)$ are also possible. These transitions are not relevant here.

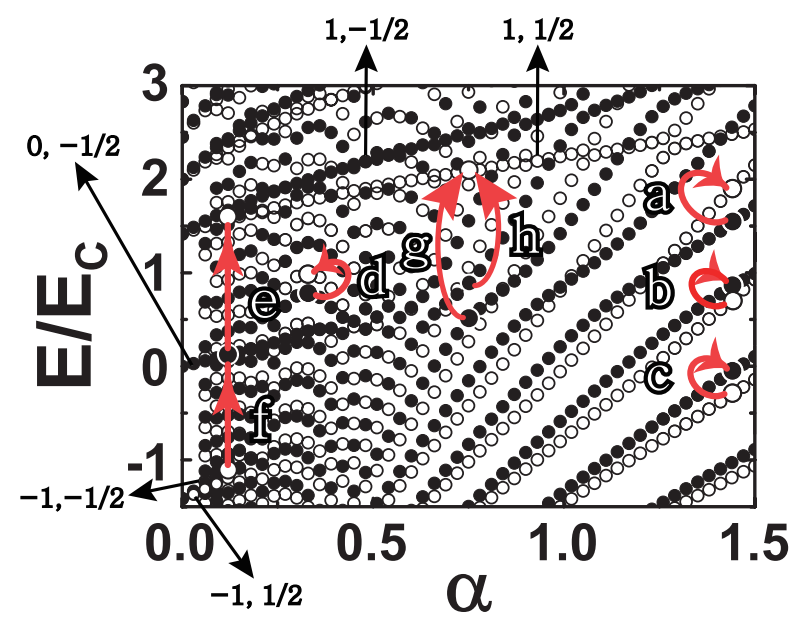

Figure 12. To display clearly possible optical transitions we plot together, for small $N_{c}=101$ and 100, the energy spectra for $J=-\frac{1}{2}$ (black dots) and $\frac{1}{2}$ (white dots). The value of the strength of the potential $\kappa=0.1 \mathrm{meV} / \mathrm{nm}^{2}$ corresponds to $\alpha=1.424$ and $\alpha=0.116$ at $B=1.5 \mathrm{~T}$ and $B=8 \mathrm{~T}$, respectively. Quasi-boundstate energies $\epsilon_{N}^{J}(\alpha)$ are labeled by $(N, J)$.

for $J=-\frac{1}{2}$ and $\frac{1}{2}$ (Results are qualitatively similar to those of a large value of $N_{c}$ ). Absorption transition strength between initial and final states $\Psi_{i}$ and $\Psi_{f}$ is $\left|\left\langle\Psi_{f}\left|\sigma_{x}\right| \Psi_{i}\right\rangle\right|^{2}$. Note that there are possible transitions from resonant quasi-boundstates of negative energies, which is a new feature. An example of this transition is shown as (f) in Fig.12, Its strength is 0.386 . Also transitions between resonant quasi-boundstates of positive energies are possible. An example is shown as (e) with the strength 0.461. Energy of a resonant quasi-boundstate will split into several values at small $B$ due to anticrossing with other levels. This will lead to a splitting of transitions. An example is shown as (g) and (h). Some examples of absorption transitions involving non-resonant states are also shown. For these transitions, due to mixing of different LL states by the 
Resonant, non-resonant, and anomalous states of Dirac electrons in a parabolic well in the presence of magn

parabolic potential, the selection rule $\Delta N= \pm 1$ must be relaxed. Examples of these transitions are shown as (a), (b), (c), and (d), in Fig.12. Their absorption strengths are 0.255 (a), 0.316(b), 0.202 (c), and 0.207 (d).

The next dominant absorption transitions occur for $\left(J, J^{\prime}\right)=(-3 / 2,-1 / 2)$. In the absence of the parabolic potential the selection rules are $\epsilon_{N}^{-3 / 2}(\alpha) \rightarrow \epsilon_{N+1}^{-1 / 2}(\alpha)$ for $N \geq 0$ and $\epsilon_{N}^{-1 / 2}(\alpha) \rightarrow \epsilon_{N+1}^{-3 / 2}(\alpha)$ for $N<0$. Similar selection rules hold for other possible $\left(J, J^{\prime}\right)$ with $\Delta J= \pm 1$.

\section{Summary}

In each Hilbert subspace of angular momentum $J$ we have studied how eigenvalues and eigenstates of a parabolic dot in a magnetic field evolve as $\alpha$ increases. We have found that they change in a non-trivial way. In the weak coupling limit of $\alpha \rightarrow 0$ one recovers discrete LL spectrum of graphene. In the intermediate coupling regime non-resonant states form a closely spaced energy spectrum, see Fig_3(a) (The result is different from the case of a cylindrical potential, whose the energy spectra in a magnetic field are discrete without quasi-boundstates [11, 14, 15] except at $B=0$ [16]). In addition, we find, counter-intuitively, that resonant quasi-boundstates of both positive and negative energies exist in the spectrum, see Fig 3(c). Closely spaced spectrum is consistent with the presence of resonant quasi-boundstates[17]. The presence of resonant quasiboundstates of negative energies is a unique property of massless Dirac fermions, but they are well-defined only for $\alpha<1$. In the strong coupling regime of $\alpha \gg 1$ all resonant and non-resonant states become anomalous states that develop a sharp peak in the well and decay slowly with small oscillations under the barrier. The average energy level spacing in each Hilbert subspace of $J$ approaches a constant value. However, note that the total density of states is the sum of each density of states computed in different Hilbert subspaces. When $\alpha$ is too large the coupling between $K$ and $K^{\prime}$ valleys may have to be included[18].

Optical transitions from resonant quasi-boundstates of positive energies are possible. There are also possible transitions from resonant quasi-boundstates of negative energies, which is a new feature. Moreover, we find that transition energies between resonant quasi-boundstates follow a scaling as a function of the coupling constant $\alpha$. Absorption transitions involving non-resonant states are also possible, and for these transitions the selection rule $\Delta N= \pm 1$ must be relaxed due to mixing of different LL states by the parabolic potential. It would be also interesting to observe experimentally the splitting of optical transition energies due to anticrossing of resonant quasi-boundstates with other states.

\section{Acknowledgments}

This research was supported by Basic Science Research Program through the National Research Foundation of Korea (NRF) funded by the Ministry of Education, Science and 
Resonant, non-resonant, and anomalous states of Dirac electrons in a parabolic well in the presence of magn

Technology (2012R1A1A2001554). We thank H. W. Lee for several useful suggestions.

\section{References}

[1] D. Heitmann and J. Kotthaus, Phys. Today 46, No.6, 56 (1993).

[2] P. L. McEuen et al., Phys. Rev. Lett. 66, 1926 (1991); J. Weis et al., Phys. Rev. B 46, 12837 (1992); T. Schmidt et al., ibid 51, 5570 (1995); S. Tarucha et al., Phys. Rev. Lett. 77, 3613 (1996); S. -R. Eric Yang, A. H. MacDonald, and M. D. Johnson, Phys. Rev. Lett. 46, 3194 (1993) ; A. H. MacDonald, S. -R. Eric Yang, M. D. Johnson, Aust. J. Phys. 46, 345 (1993) .

[3] V. Fock, Z. Phys. 47, 446-448 (1928); C. G. Darwin, Proc. Camb. Philos. Soc. 27, 86-90 (1930).

[4] A. K. Geim and A. H. MacDonald, Phys. Today 60, 35 (2007); A. H. Castro Neto et al., Rev. Mod. Phys., 81, 109 (2009).

[5] G. Giavaras, P. A. Maksim, and M. Roy, J. Phys.: Condens. Matter 21, 102201 (2009). This paper contains interesting investigation of confinement and definement transitions in various potentials.

[6] H. Y. Chen, V. Apalkov, and T. Chakraborty, Phys. Rev. Lett. 98, 186803 (2007). For a Comment on this paper see Ref. [7].

[7] P. S. Park, S. C. Kim, and S. -R. Eric Yang, Phys. Rev. Lett. 108, 169701 (2012).

[8] C. Bender and S. Orszag, Advanced Mathematical Methods for Scientists and Engineers (McGraw Hill, New York, 1978).

[9] Similar problem is investigated for an impurity in an ordinary semiconductor from zero to finite magnetic fields; see A. H. MacDonald and D. S. Ritchie, Phys. Rev. B 33, 8336 (1986).

[10] D. Yoshioka, The Quantum Hall Effect (Springer, Berlin, 1998).

[11] P. S. Park, S. C. Kim, and S. -R. Eric Yang, Phys. Rev. B 84, 085405 (2011).

[12] In the strong coupling limit many levels with different values of $n$ are heavily mixed.

[13] For sufficiently large $\alpha$ even eigenstates with eigenvalues close to zero have narrow probability densities near $r=0$.

[14] P. S. Park, S. C. Kim, and S. -R. Eric Yang, J. Phys.; Condens. Matter 22, 375302 (2010); S. C. Kim and S. -R. Eric Yang, J. Phys.: Condens. Matter 24195301 (2012). Properties of a single antidot and an array of periodic antidots are investigated in these papers.

[15] P. Recher, J. Nilsson, G. Burkard, B. Trauzettel, Phys. Rev. B 79, 085407 (2009).

[16] A. Matulis and F. M. Peeters, Phys. Rev. B 77, 115423 (2008).

[17] Quantum mechanics, L. D. Landau and L. Lifshitz (3rd ed., Pergamon Press, Oxford, 1977).

[18] J. W. Lee, S. C. Kim, and S. -R. Eric Yang, Solid State Commun. 152, 1929 (2012). Ferromagnetism of one-dimensional electron gas of an armchair ribbon is investigated. 